\title{
IFMIF suitability for evaluation of fusion functional materials
}

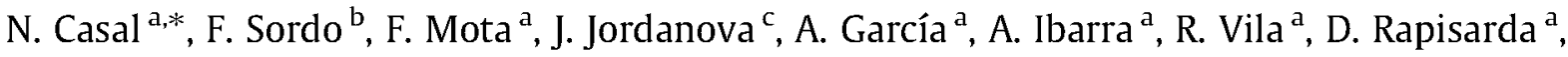 \\ V. Queral ${ }^{\mathrm{a}}$, M. Perlado ${ }^{\mathrm{a}}$ \\ ${ }^{a}$ Laboratorio Nacional de Fusión, EURATOM-CIEMAT, 28040 Madrid, Spain \\ ${ }^{\mathrm{b}}$ Universidad Politécnica de Madrid, 28006 Madrid, Spain \\ ${ }^{c}$ Institute for Nuclear Research and Nuclear Energy, 1784 Sofia, Bulgaria
}

\begin{abstract}
A B S T R A C T
The International Fusion Materials Irradiation Facility (IFMIF) is a future neutron source based on the D-Li stripping reaction, planned to test candidate fusion materials at relevant fusion irradiation conditions. During the design of IFMIF special attention was paid to the structural materials for the blanket and first wall, because they will be exposed to the most severe irradiation conditions in a fusion reactor. Also the irradiation of candidate materials for solid breeder blankets is planned in the IFMIF reference design.

This paper focuses on the assessment of the suitability of IFMIF irradiation conditions for testing functional materials to be used in liquid blankets and diagnostics systems, since they are been also considered within IFMIF objectives. The study has been based on the analysis and comparison of the main expected irradiation parameters in IFMIF and DEMO reactor.
\end{abstract}

\section{Introduction}

The future International Fusion Materials Irradiation Facility (IFMIF) facility will be a special irradiation tool for the qualification of promising materials for fusion reactors [1]. To fulfil this objective, IFMIF must meet the fusion neutron spectrum as closely as possible to reach the displacement damage per atom (dpa), primary recoil spectrum (PKA) and gaseous elements by transmutation reactions $(\mathrm{He}, \mathrm{H})$ produced in the fusion DEMO reactors. The High Flux Test Module (HFTM) of IFMIF (up to $10^{15} \mathrm{n} / \mathrm{cm}^{2} / \mathrm{s}$ fluence rate and 50 dpa per full power year), is aimed at the irradiation of candidate structural materials for DEMO reactors. Gas generation and PKA spectrum in that zone are close to the expected values in structural materials of DEMO reactor [2]. The irradiation of candidate materials for solid breeder blankets is also planned in the IFMIF design [3,4] since the irradiation in the Medium Flux Test Modules (MFTM) has been found more suitable to match the fusion irradiation environment than irradiation conditions in fission reactors [5].

Liquid metal blankets are a promising blanket option since they allow high operating temperature, adequate tritium breeding without a beryllium neutron multiplier, easy maintenance and low pumping power [6,7]. Nevertheless, several problems associated with them include magneto-hydrodynamic (MHD) effects, tritium permeation and control issues, and liquid-structural material compatibility. Materials selection plays a significant role in minimizing these problems. For example, the use of special coatings (insulating, antipermeation, and anticorrosion) such as $\mathrm{Al}_{2} \mathrm{O}_{3}$, AlN, $\mathrm{CaO}$, or others, could be decisive for the feasibility of the several designs [8]. On the other hand, the blanket components will be exposed to $14 \mathrm{MeV}$ neutrons with high intensities that will produce nuclear transmutation atoms and atomic displacement cascades. These processes can have an important impact on the materials properties and long term activation.

For the materials in diagnostic systems, the high radiation level in DEMO will prevent the use of many of the diagnostics planned for the International Thermonuclear Experimental Reactor (ITER). Practically all diagnostics systems use ceramics as electrical insulation, making them key materials for acceptable system development. Also, the materials to be used as transmission components in windows (such as $\mathrm{SiO}_{2}$ or $\mathrm{Si}_{3} \mathrm{~N}_{4}$ ) will be exposed to very high radiation fields [9].

Several types of degradation phenomena have been identified for these different materials and are being investigated since they affect the structural properties, the tritium transport and other properties that enable the special function of each material. Degradation effects can include radiation-induced conductivity (RIC) or radiation-induced electrical degradation (RIED) in insulating materials, effects of hardening, swelling, liquid-metal embrittlement in materials in contact with the liquid breeder, radiation induced optical absorption in transmission materials, surface degradation 
or radiation-corrosion interactions, among other important effects, widely studied in recent years [10].

The maximum neutron fluence limit in ITER is too low to fully evaluate the effects of a significant and continuous neutron irradiation on materials [11], but in situ measurements in experiments in a relevant irradiation environment will provide important data for these issues.

The objective of this work is to assess the suitability of IFMIF to perform relevant tests with functional materials for liquid breeder blankets and diagnostics components of fusion reactors. Neutronic calculations have produced the main irradiation parameters for different positions of IFMIF. The results are compared with the expected values for a helium cooled lead lithium (HCLL) DEMO reactor [12] to define the best configuration to irradiate these materials in IFMIF. The materials considered are $\mathrm{Fe}, \mathrm{SiC}, \mathrm{CaO}, \mathrm{Al}_{2} \mathrm{O}_{3}, \mathrm{AlN}$, $\mathrm{SiO}_{2}$ and $\mathrm{Si}_{3} \mathrm{~N}_{4}$.

\section{Neutronic calculations}

Calculations have been performed to obtain the displacement damage (dpa) and gas to dpa ratios as initial indicators of behaviour under irradiation of the selected materials in different zones of IFMIF (the high flux and Medium Flux irradiation zones). These parameters have been also calculated for first wall and breeder zone of DEMO HCLL, and for a typical high flux fission reactor (HFR).

It is well known that different primary recoil energy spectra can produce completely different damage morphologies; therefore these spectra should be taken into account for each material in a future extensive assessment program.

The irradiation parameters were calculated using MCNPX [13] considering all the reaction processes included in the ENDF/B-VII library with values for neutron reaction cross sections up to $150 \mathrm{MeV}$ for the nuclei considered. The neutron spectrum (VITAMIN-J 211 energy group structure) of each facility and position has been used to simulate irradiation of the material of interest. Each spectrum has been used as neutron source in the center of a spherical sample small enough to accurately reproduce irradiation. To obtain the dpa for each material, weighted average damage threshold energy (Ed) has been considered [2,14-19]. Table 2 shows these values for each element.
The spectra have been obtained, in the case of IFMIF, by means of McDelicious code, developed by FZK (Forchungszentrum Karlsruhe, Germany) on the basis of MCNP code [20] for computations of the IFMIF Test Cell, to accurately simulate the neutrons source. The positions evaluated are shown in Fig. 1 (positions from 1 to 5$)$.

Positions 2 and 3 correspond, respectively, to the Medium Flux region behind the Moderator Module (that is the TRM reference position) and the Medium Flux region just behind the HFTM (that is the CFTM reference position). For the calculations of these two spectra, the model of the Test Cell originally included in the code has been modified by filling the eight rigs of the TRM with LiPb.

The possibility of testing in the High Flux region has been also considered. The spectrum of one of the rigs of the HFTM reference design has been used in the calculations, and this represents the position 1. In this case, the reference McDelicious model has been used for calculating the spectrum.

Also a possible extension of the current HFTM has been taken into account by considering an internal companion rig (ICR, position 4) and the following external companion rig (ECR, position 5).

Calculations for all these positions used the spectra averaged over the whole corresponding rig.

For the DEMO HCLL, spectra corresponding to $4000 \mathrm{MW}$ of fusion power have been used in the calculations [21]. Four locations of an inboard blanket have been selected: the front of the first wall (FW), the back of the first wall, a middle point of the breeder zone (BZ) and the back of the breeder zone.

For comparison with a fission reactor, a typical spectrum for a high flux fission reactor (HFR) has been used [3].

\section{Results and discussion}

The calculated values of dpa and hydrogen and helium production are shown in Table 1 for the materials in the different positions. The results show that in IFMIF, as expected, for all the materials analyzed, as you move away from the HFTM along the beam direction, lower dpa values are obtained. With respect to the newly considered companion rigs, in the case of the ICR (position 4) the calculated dpa fall between the values in positions 3 and 2. In the case of the ECR (position 5), similar or slightly lower
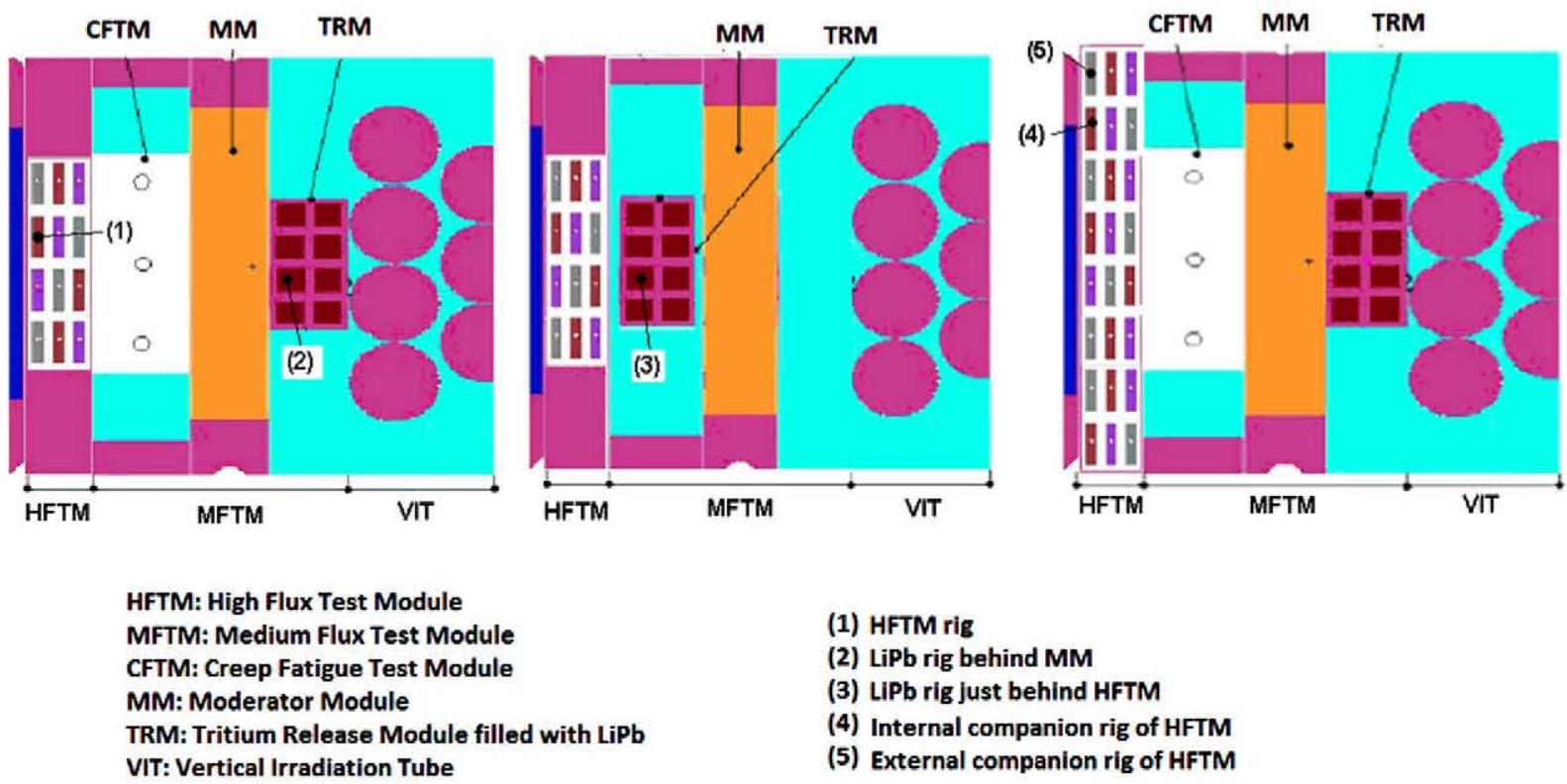

(1) HFTM rig

(2) LiPb rig behind MM

(3) LIPb rig just behind HFTM

(4) Internal companion rig of HFTM

(5) External companion rig of HFTM

Fig. 1. IFMIF geometric model used with McDelicious calculations. Positions considered (from 1 to 5 ) are indicated. 
Table 1

Calculated irradiation parameters for all materials considered.

\begin{tabular}{|c|c|c|c|c|c|c|c|c|c|c|}
\hline \multirow[t]{2}{*}{ dpa/fpy, appm/fpy } & \multicolumn{4}{|c|}{ DEMO HCLL (4000 MW) } & \multicolumn{5}{|l|}{ IFMIF } & \multirow{2}{*}{$\begin{array}{l}\text { HFR } \\
\text { Typical spectrum }\end{array}$} \\
\hline & FW (front) & FW (back) & BZ (middle) & BZ (back) & $\operatorname{HFTM}(1)$ & MFTM (2) & MFTM (3) & $\operatorname{ICR}(4)$ & ECR (5) & \\
\hline \multicolumn{11}{|l|}{$\mathrm{Fe}-56$} \\
\hline dpa & 30 & 29 & 8 & 2 & 31 & 2 & 13 & 7 & 2 & 4 \\
\hline $\mathrm{H}$ & 982 & 870 & 53 & 4 & 1329 & 87 & 605 & 201 & 33 & 3 \\
\hline $\mathrm{He}$ & 270 & 241 & 16 & 1 & 402 & 26 & 180 & 62 & 11 & 1 \\
\hline \multicolumn{11}{|l|}{$\mathrm{SiC}$} \\
\hline dpa & 20 & 20 & 8 & 3 & 15 & 2 & 6 & 4 & 1 & 3 \\
\hline $\mathrm{H}$ & 1053 & 939 & 62 & 5 & 1330 & 85 & 589 & 208 & 37 & 11 \\
\hline $\mathrm{He}$ & 2596 & 2304 & 144 & 11 & 2707 & 178 & 1230 & 408 & 70 & 9 \\
\hline \multicolumn{11}{|l|}{$\mathrm{SiO}_{2}$} \\
\hline $\begin{array}{l}\text { apa } \\
\mathrm{H}\end{array}$ & $\begin{array}{r}48 \\
929\end{array}$ & $\begin{array}{r}49 \\
827\end{array}$ & $\begin{array}{l}21 \\
53\end{array}$ & $\begin{array}{l}8 \\
4\end{array}$ & $\begin{array}{r}34 \\
1182\end{array}$ & $\begin{array}{r}4 \\
77\end{array}$ & $\begin{array}{r}14 \\
530\end{array}$ & $\begin{array}{r}9 \\
183\end{array}$ & 32 & $\begin{array}{l}8 \\
7\end{array}$ \\
\hline $\mathrm{He}$ & 1477 & 1319 & 87 & 7 & 1709 & 108 & 752 & 270 & 50 & 21 \\
\hline \multicolumn{11}{|l|}{$\mathrm{Al}_{2} \mathrm{O}_{3}$} \\
\hline dpa & 19 & 20 & 9 & 3 & 14 & 2 & 5 & 4 & 1 & 3 \\
\hline $\mathrm{H}$ & 1114 & 987 & 60 & 4 & 1119 & 74 & 511 & 169 & 28 & 5 \\
\hline $\mathrm{He}$ & 1290 & 1150 & 75 & 6 & 1458 & 93 & 645 & 228 & 42 & 17 \\
\hline \multicolumn{11}{|l|}{$\mathrm{Si}_{3} \mathrm{~N}_{4}$} \\
\hline $\mathrm{dpa}$ & 17 & 17 & 7 & 3 & 13 & 1 & 5 & 3 & 1 & 3 \\
\hline $\mathrm{H}$ & 2511 & 2339 & 398 & 117 & 2360 & 169 & 1027 & 424 & 104 & 1942 \\
\hline $\mathrm{He}$ & 1287 & 1207 & 150 & 17 & 1881 & 116 & 768 & 356 & 85 & 183 \\
\hline \multicolumn{11}{|l|}{$\mathrm{CaO}$} \\
\hline $\mathrm{dpa}$ & 17 & 17 & 7 & 3 & 14 & 1 & 6 & 4 & 1 & 3 \\
\hline $\mathrm{H}$ & 2975 & 2698 & 215 & 18 & 3847 & 242 & 1639 & 653 & 134 & 175 \\
\hline $\mathrm{He}$ & 1475 & 1335 & 103 & 8 & 1964 & 122 & 835 & 331 & 67 & 78 \\
\hline \multicolumn{11}{|l|}{$A I N$} \\
\hline dpa & 21 & 21 & 9 & 3 & 16 & 2 & 6 & 4 & 1 & 4 \\
\hline $\mathrm{H}$ & 2545 & 2350 & 363 & 104 & 2132 & 154 & 941 & 375 & 90 & 1697 \\
\hline $\mathrm{He}$ & 1076 & 1011 & 127 & 14 & 1545 & 96 & 632 & 294 & 70 & 157 \\
\hline
\end{tabular}

values of dpa than in position 2 are found. Roughly the same effects have been found for all materials for the gas production reactions.

Nevertheless, for the evaluation of the effects of transmutation gases in materials, usually ratios of helium (appm/fpy) and hydrogen (appm/fpy) production to displacement damage (dpa/fpy) are used and are the main indicators used in this assessment. Fig. 2 show the ratio of $\mathrm{H}$ to dpa versus the ratio of He to dpa in some of these materials. The values of ratios obtained for DEMO are enclosed by a shaded ellipse, rhombi represent the calculated values for the different positions in IFMIF and the triangles indicate the ratios for the HFR.

$\mathrm{Al}_{2} \mathrm{O}_{3}, \mathrm{SiO}_{2}, \mathrm{CaO}$ and $\mathrm{SiC}$ can be jointly analyzed since they show similar behaviour. The calculated ratios of these materials show that position 2 in the Medium Flux region and position 4 (ICR) best fit most irradiated positions of DEMO, while positions 1 and 3

Table 2

Damage threshold energy (Ed) for each element.

\begin{tabular}{lll}
\hline Compound & Element & Ed (eV) \\
\hline $\mathrm{Fe}$ & $\mathrm{Fe}$ & 40 \\
$\mathrm{SiC}$ & $\mathrm{Si}$ & 70 \\
& $\mathrm{C}$ & 38 \\
$\mathrm{Al}_{2} \mathrm{O}_{3}$ & $\mathrm{Al}$ & 34 \\
& $\mathrm{O}$ & 83 \\
$\mathrm{SiO}_{2}$ & $\mathrm{Si}$ & 35 \\
& $\mathrm{O}$ & 20 \\
$\mathrm{Si}_{3} \mathrm{~N}_{4}$ & $\mathrm{Si}$ & 60 \\
& $\mathrm{~N}$ & 60 \\
$\mathrm{CaO}$ & $\mathrm{Ca}$ & 65 \\
& $\mathrm{O}$ & 50 \\
$\mathrm{AIN}$ & $\mathrm{Al}$ & 50 \\
& $\mathrm{~N}$ & 50 \\
\hline
\end{tabular}

show higher values of ratios than DEMO (as seen in Fig. 2 for results of $\mathrm{SiO}_{2}$ and $\mathrm{SiC}$ ). In contrast, position 5 show ratios similar to the middle zone of the DEMO breeder. It should be emphasized that irradiation of these materials in position 4 gives the advantage of almost three times higher damage rate than in position 2 . The results for HFR show that $\mathrm{He}$ and $\mathrm{H}$ per dpa ratios are too low to simulate the DEMO breeder zone for $\mathrm{SiC}, \mathrm{SiO}_{2}$ and $\mathrm{Al}_{2} \mathrm{O}_{3}$. In the case of $\mathrm{CaO}$, the values are slightly higher to those expected in the center of the breeder zone.

For $\mathrm{Si}_{3} \mathrm{~N}_{4}$ and $\mathrm{AlN}$ (see AlN in Fig. 2) the results show that in IFMIF positions 2 and 4 , which have been found to be the more suitable for testing the oxides and $\mathrm{SiC}$, the ratios $\mathrm{H} / \mathrm{dpa}$ also match most irradiated zones of DEMO reactor, but a comparatively higher He production ratio than $\mathrm{H}$ production ratio with respect to the DEMO values is found. This effect is particularly significant in position 4. For these two materials the $\mathrm{H}$ production ratio is typical of the DEMO breeder zone while the He production ratio fits the FW location. For HFR the production of $\mathrm{H}$ is higher than in the other materials, due to the contribution of the low energy part of the HFR spectrum. Nevertheless, for the He production, a ratio typical of the front part of the breeder zone is expected.

$\mathrm{Fe}-56$ has been also studied since coatings of $\mathrm{Al}_{2} \mathrm{O}_{3}, \mathrm{AlN}$ or $\mathrm{CaO}$ may be used on the Fe-based structures of the breeder zone. In this case, damage production in the HFTM agrees with the result obtained for the first wall of DEMO, as reported earlier [22,23]. Values of gas production ratios in HFTM are also very similar to the first wall of DEMO reactor, although slightly higher. In position 2 of Medium Flux region values of gas ratios fit those expected for the first wall. This is a particularly interesting result since this position is also suitable to irradiate the coating materials.

It has to be noted that when selecting a "suitable" position to test the materials to be used as insulating coatings, such as $\mathrm{Al}_{2} \mathrm{O}_{3}, \mathrm{AlN}$ or $\mathrm{CaO}$, the role of $\mathrm{H}$ is very important. At elevated 


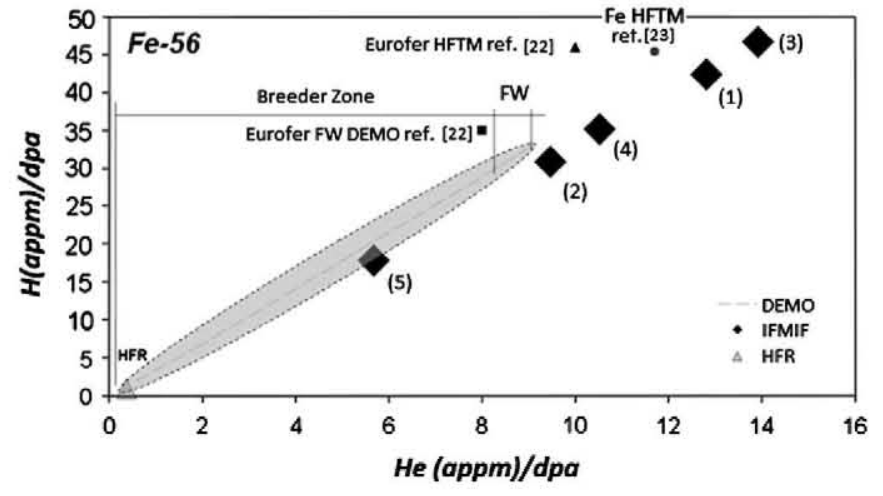

(a)

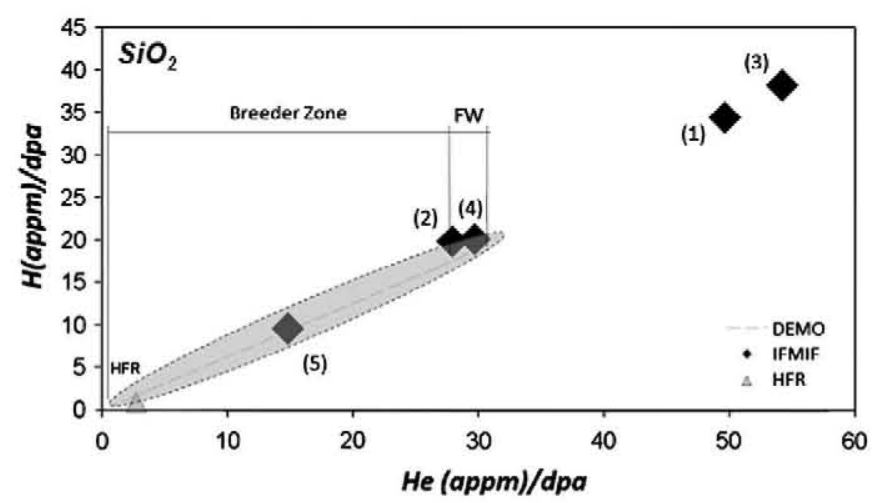

(c)

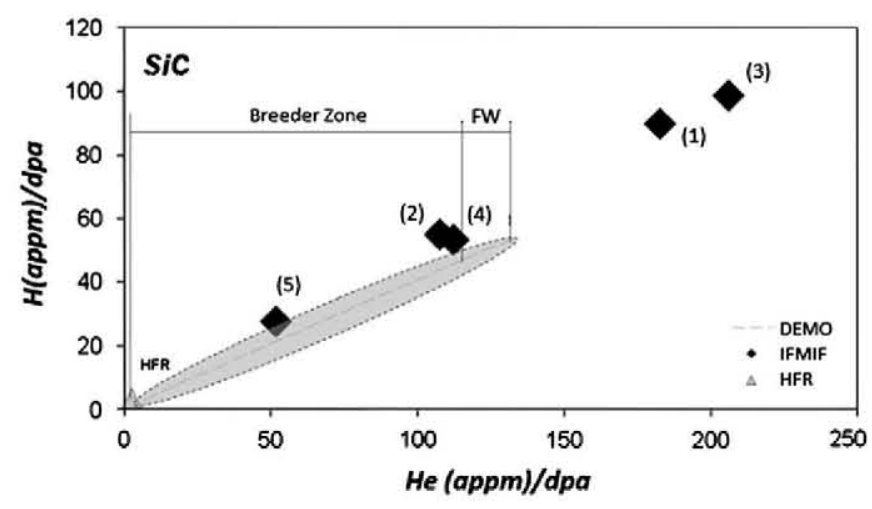

(b)

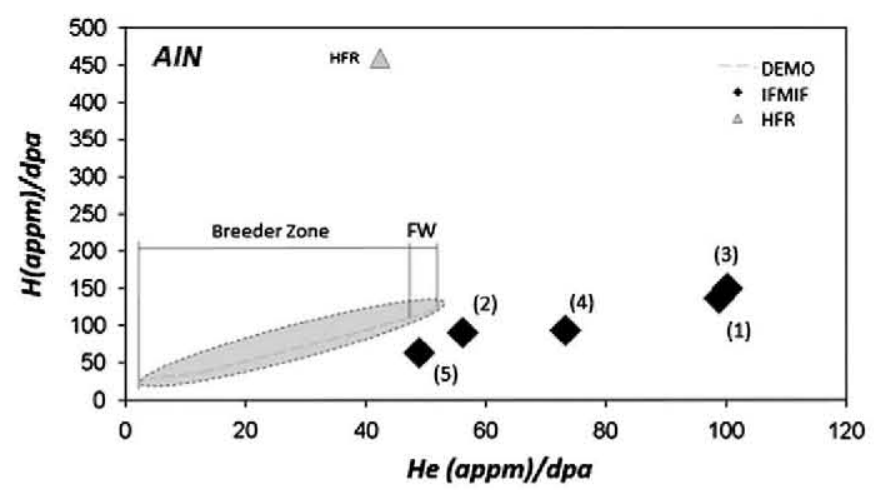

(d)

Fig. 2. Gas transmutation ratios for $\mathrm{Fe}-56, \mathrm{SiC}, \mathrm{SiO}_{2}$ and $\mathrm{AIN}$.

temperatures, the high concentrations of this gas will increase the ionic conductivity. For testing these materials then, position 2 is suitable, since the amount of $\mathrm{H}$ per dpa corresponds to the expected value for a high irradiation zone of the DEMO HCLL blanket. The He production is also adequate, and that is important since $\mathrm{H}$ behaviour could change when produced simultaneously with $\mathrm{He}$. Irradiation in the internal companion rig also gives high dpa production rates in these materials. For AlN, irradiation in this rig implies that higher concentrations of He are obtained.

It is important that accelerated testing of these materials is possible using positions 1 or 3 , in which damage production is very high, but it implies increasing the $\mathrm{He}$ and $\mathrm{H}$ ratios per $\mathrm{dpa}$. The particular case of $\mathrm{Si}_{3} \mathrm{~N}_{4}$ or AlN in which only the He/dpa is increased, is also noted.

\section{Conclusions}

IFMIF will be a dedicated irradiation facility that will provide high availability to test candidate fusion reactor materials. A first analysis of neutron response of functional materials for liquid breeder blankets and diagnostics has been performed in order to asses the suitability of irradiation of these materials in IFMIF. The following conclusions have been obtained.

(1) TRM reference position 2 is a suitable position in IFMIF for testing functional materials, including $\mathrm{SiC}, \mathrm{SiO}_{2}, \mathrm{Al}_{2} \mathrm{O}_{3}$, $\mathrm{Si}_{3} \mathrm{~N}_{4}$ and $\mathrm{AlN}$, since the most important responses (ratio of helium to dpa and ratio of hydrogen to dpa) are very close to the $4000 \mathrm{MW}$ DEMO HCLL in the highest irradiation zones. In addition, the tritium breeding ratio expected in this position [24] is representative of the breeder zone of a HCLL blanket, which is especially significant for the functional materials that may be used in the DEMO breeder zone.

(2) The irradiation of $\mathrm{SiC}, \mathrm{SiO}_{2}, \mathrm{Al}_{2} \mathrm{O}_{3}$ or $\mathrm{CaO}$ in the internal companion rig of an extended HFTM (position 4) provides the advantage of accelerating testing with respect to position 2 , since higher damage is obtained while maintaining the proper gas ratios. In the case of $\mathrm{Si}_{3} \mathrm{~N}_{4}$ and $\mathrm{AlN}$ a higher value of $\mathrm{He} / \mathrm{dpa}$ is obtained with respect to the H/dpa.

(3) In the high flux fission reactor, the ratios of helium to dpa and hydrogen to dpa are very close to the expected values at the back of the DEMO HCLL breeder zone for all these materials, except for $\mathrm{N}$ containing compounds, in which a huge amount of hydrogen is generated.

(4) The external companion rig (position 5) of an extended HFTM provides neutron responses similar to that expected in the center of the DEMO HCLL breeder zone for most of the studied materials.

(5) Irradiations in HFTM (1) and position 3 of Medium Flux region provide significant dpa, very close to the expected values in the highest irradiation zones of DEMO HCLL, although slightly lower.

\section{References}

[1] IFMIF International Team, IFMIF Comprehensive Design Report, 2004.

[2] S.P. Simakov et al., J. Nucl. Mater. 386-388 (2009) 52-55.

[3] A. Moeslang, P. Vladimirov, Fusion Eng. Des. 63-64 (2002) 121-126.

[4] A. Moeslang, Irradiation parameters of Beryllium Under IFMIF Irradiation Conditions, Final Report on the EFDA Task TW3-TTMI-003-D8, 2004

[5] U. Fischer, Nuclear irradiation parameters of beryllium under fusion, fission and IFMIF irradiation conditions, in: Proceedings of 6th IEA Workshop on Beryllium Technology for Fusion, Miyazaki, Japan, 2003 (December). 
[6] D. Maisonnier, A Conceptual Study of Commercial Fusion Power Plants, Final Report of the European Fusion Power Plant Conceptual Study (PPCS), 2005 (April).

[7] M. Malang, Effective Power Management in DEMO: Work Necessary to Prove Feasibility and to Increase Attractiveness, ARIES Project Meeting, 2007 (September).

[8] S. Malang et al., Fusion Eng. Des. 27 (1995) 570-586.

[9] M. Decreton, Tatsuo Shikama, Eric Hodgson, J. Nucl. Mater. 329-333 (2004) 125-132.

[10] E.R. Hodgson, Nucl. Instr. Methods 191 (2002) 744-751.

[11] S. Malang, A.R. Raffray, N.B. Morley, Fusion Eng. Des. 84 (2009) 2145-2157.

[12] A. Li Puma, Helium-cooled Lithium-lead Fusion Power Plant (PPCS Model AB) Design and Integration of In-vessel Components and Associated Systems, EFDA Task TW4-TRP-002-D04, 2005
13] 〈http://mcnpx.lanl.gov>.

[14] L. Malerba, J.M. Perlado, Phys. Rev. B 65 (2002) 4.

[15] J.B. Wachtman, Ceramic Films and Coating, Noyes Publications, 1993.

[16] G.W. Arnold, W.D. Compton, Phys. Rev. 4 (1960) 2.

[17] F. Mota et al., Fusion Eng. Des. 75-79 (2005) 1027-1030.

[18] J.L. Grant, R. Cooper, J. Phys. Chem. 88 (1988) 4158.

19] P.V. Vladimirov et al., J. Nucl. Mater. 253 (1998) 104-112.

20] S.P. Simakov et al., J. Nucl. Mat. 307-311 (2002) 1710-1714.

[21] Jordana Jordanova, Institute for Nuclear Research and Nuclear Energy in Sofia, Bulgary, personal communication.

[22] U. Fischer, S.P. Simakov, P.P.H. Wilson, J. Nucl. Mater. 329-333 (2004) 228 232.

[23] E. Daum, J. Nucl. Mater. 283-287 (2000) 1001-1005

[24] N. Casal et al., Fusion Eng. Des. 84 (2009) 559-564. 